\title{
RESUMEN DE LA ACTIVIDAD SÍSMICA Y VOLCÁNICA EN COSTA RICA DURANTE EL AÑO 2010
}

\author{
SUMMARY OF THE SEISMIC AND VOLCANIC ACTIVITY IN COSTA RICA \\ DURING 2010
}

\author{
Rafael Barquero ${ }^{1} \&$ Wilfredo Rojas ${ }^{2}$ \\ Red Sismológica Nacional (RSN-UCR-ICE) \\ ${ }^{1}$ Área de Amenazas y Auscultación Sísmica y Volcánica, Exploración \\ Subterránea, PySA, ICE; Apdo. 10032-1000 San José. \\ ${ }^{2}$ Sección de Sismología y Vulcanología. Escuela Centroamericana de Geología (UCR) \\ *Autor para contacto: RbarqueroP@ice.go.cr
}

\begin{abstract}
Durante el año 2010 la Red Sismológica Nacional (RSN) ubicó alrededor de 3000 sismos dentro del territorio nacional (Fig. 1) de los cuales 111 fueron reportados como sentidos por la población de Costa Rica, actividad similar a la del 2009, año en que fueron reportados 115 eventos sentidos. 49 de los sismos sentidos tuvieron magnitudes menores a 4,0 y 62 magnitudes mayores o iguales a 4,0. Los sismos de mayor magnitud en nuestro país en el 2010 fueron el sismo del 20 de mayo que se ubicó $25 \mathrm{~km}$ SSW de Quepos de magnitud 5,9 asociado al proceso de subducción de la placa Coco y el sismo de Zarcero del 8 de octubre, el cual tuvo una magnitud de 5,9 y una profundidad de $84 \mathrm{~km}$ (sismo intraplaca). Estos temblores aunque se sintieron fuertes no produjeron serios daños. De estos sismos 53 (47\%) fueron sismos superficiales (prof. $<20 \mathrm{~km}$ ) originados por fallas locales. El proceso de subducción de las placas
\end{abstract}

de Coco bajo la placa Caribe y la Microplaca de Panamá y algunas rupturas en fallas más profundas fueron responsable del $53 \%$ restante que contabilizó 58 eventos.

En cuanto a la actividad volcánica, fue relevante durante el 2010 la intensificación de la actividad en el volcán Turrialba y algunos flujos piroclásticos en el volcán Arenal.

\section{Sismos reportados como sentidos}

En el 2010 las estaciones de la RSN registraron 111 temblores reportados como sentidos por la población (Figs. 2 y 3), que por lo general son los de mayor magnitud entre los cientos de sismos que registra la RSN mensualmente. De ellos, 58 tuvieron origen en el proceso de subducción de la placa del Coco bajo la placa Caribe, por lo que 


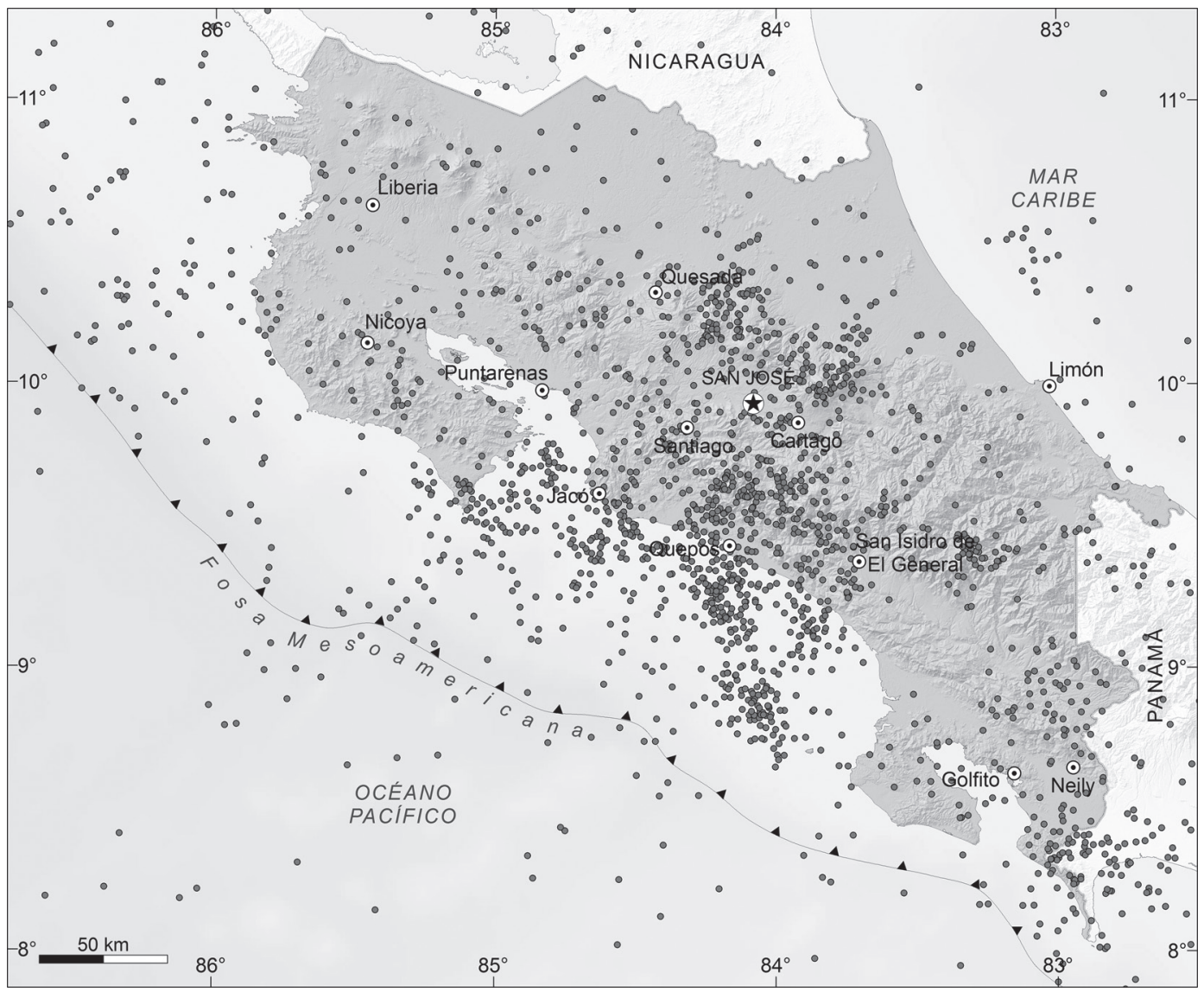

Fig. 1: Mapa de los sismos registrados y localizados durante el año 2010 por la RSN.

son sismos generalmente de profundidad intermedia; otros 53 se asocian con fallamiento local. Los meses con mayor cantidad de sismos sentidos fueron enero, marzo, julio y setiembre con 31,11 , 11 y 16 eventos respectivamente, y los meses con menos eventos fueron junio y diciembre con solamente 2 y 3 eventos.

La actividad sísmica más alta durante el año 2010 fue en el mes de enero, a raíz principalmente de un enjambre sísmico en la zona sur. Luego tendió a decaer los meses siguientes, pero en setiembre se inició otro repunte importante, pero en fuentes sísmicas diferentes, cuando se registraron 16 sismos sentidos principalmente localizados en el extremo sur de la península de Nicoya y al norte de Buenos Aires de Osa.

\section{Magnitudes de los sismos sentidos}

La distribución por rangos de las magnitudes de los sismos sentidos registrados en el año 2010 se muestra en el cuadro 1. Durante el año ocurrieron sismos de magnitud hasta 5.9 dentro o cerca 


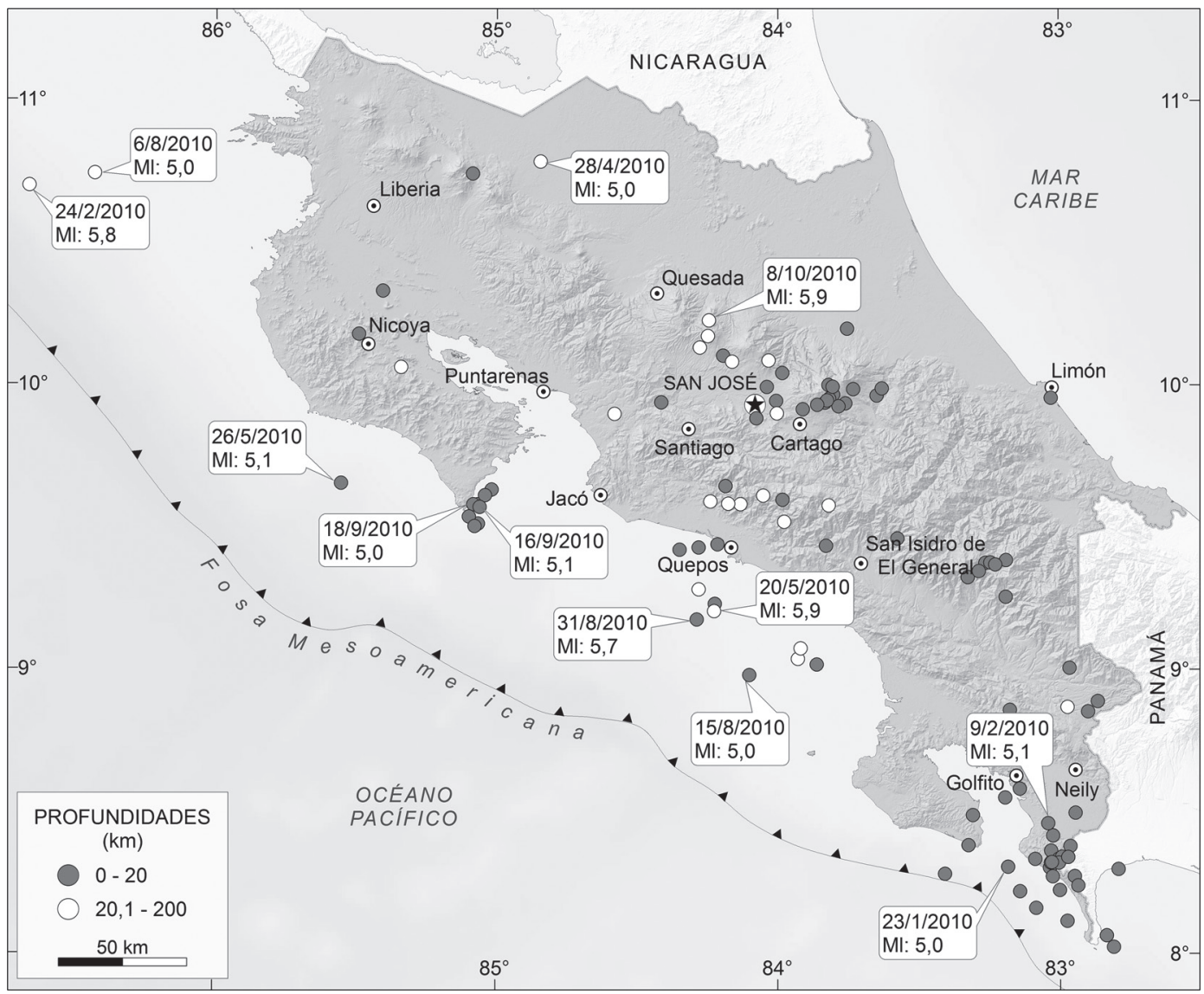

Fig. 2: Mapa de los sismos reportados como sentidos durante el 2010 en Costa Rica.

de nuestro país y la mayoría de los eventos son de magnitudes moderadas (entre 3 y 4,9). Se registraron 14 eventos con magnitudes mayores a 5,0 y menores a 6,0 (Fig. 2). El gráfico 2 muestra los sismos con sus magnitudes durante el año 2010.

\section{Profundidades de los sismos sentidos}

De los 111 temblores ocurridos en el año 201053 son de foco superficial $(0-20 \mathrm{~km})$ y se deben a fallas geológicas desestabilizadas por las fuerzas tectónicas. Los otros 58 se atribuyen a la subducción de la placa del Coco bajo la placa Caribe. Estos sismos de profundidad intermedia y profunda se concentraron principalmente en el sector central del país y algunos en el pacífico norte (Guanacaste) . En la figura 4 se puede observar que la mayor parte de los sismos sentidos son de profundidades menores a $20 \mathrm{~km}$. Hubo varios de gran profundidad (mayor a $80 \mathrm{~km}$ ) como los sismos que ocurrieron en el mes de octubre al noreste de Zarcero. El sismo con una profundidad de $138 \mathrm{~km}$ ocurrió el 28 de abril, tuvo una 
Cuadro 1

Distribución de magnitudes de los sismos sentidos en Costa Rica durante el 2010

\begin{tabular}{cc}
\hline Rango & No. sismos \\
\hline $2,0-2,9$ & 4 \\
$3,0-3,9$ & 45 \\
$4,0-4,9$ & 47 \\
$5,0-5,9$ & 14 \\
\hline
\end{tabular}

magnitud de 5,0 y se localizó bajo San Rafael de Guatuso y por ser tan profundo no produjo daños en esa zona.

\section{FUENTES SÍSMICAS PRINCIPALES}

\section{Proceso de subducción de la placa del Coco bajo la placa Caribe}

La interacción de las placas Coco-Caribe fue más intensa en la región de la zona sur en donde se registró varios temblores importantes. Entre los días sábado 23 y el lunes 25 de enero de 2010 , se registraron unos 60 sismos en la zona sur de Costa Rica y el suroeste de Panamá, destacando un total de 32 sismos con magnitudes mayores a 3 en la escala Richter, que son los mayormente sentidos por la población de la zona. Estos se ubicaron en la zona de la Península Burica, siendo debidos a procesos tectónicos dentro de la región del Punto Triple de interacción entre las placas, Coco, Nazca y Bloque de Panamá. Entre éstos sobresalen los dos más fuertes, sucedidos el día 23; el primero, ocurrido a las 03:09 am de magnitud local escala Richter 5,0 Ml y el otro a las 08:43 am de magnitud 4,9 Ml. Parte de estos eventos tienen epicentros frente a la costa oeste de la Península Burica y otros ubicados en la parte central de esa Península.

En segundo lugar de importancia estuvo la actividad sísmica del Pacífico Central, entre
Herradura y Dominical donde se ubicaron 15 de los sismos sentidos del año 2010 y la sismicidad asociada a esta fuente no superó magnitudes mayores a 5,0 .

La región de Guanacaste tuvo poca actividad por la subducción, se localizaron solo 5 eventos en esta región. Un sismo profundo (prof. $138 \mathrm{~km}$ ) se produjo el día 27 de abril en San Rafael de Guatuso, este sismo fue de magnitud 5,0 y fue sentido en esa zona en forma leve dada su gran profundidad.

En la región de la Cordillera Volcánica Central se localizaron 6 eventos profundos. El sismo principal de esta serie tuvo una magnitud de 5,9 (Mw), se ubicó al NE de Zarcero y fue muy profundo $(84 \mathrm{~km})$ y fue seguido por varias réplicas. Esta actividad se relaciona con una ruptura profunda en la placa del Coco y se le denomina sismos intraplaca.

\section{Fallamiento cortical}

De los 53 sismos superficiales originados por fallas locales que fueron eventos sentidos las zonas en donde se registró mayor actividad fue principalmente fallas en las zonas de Cóbano, norte de Buenos Aires, macizos Irazú y Turrialba, Sabanilla, Desamparados, Zona de los Santos, norte de San Isidro de Pérez Zeledón y San Vito de Coto Brus.

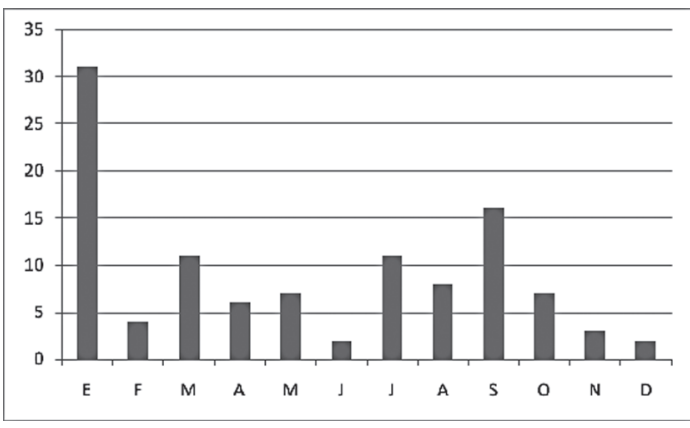

Fig. 3: Número de sismos sentidos por mes durante el año 2010. 


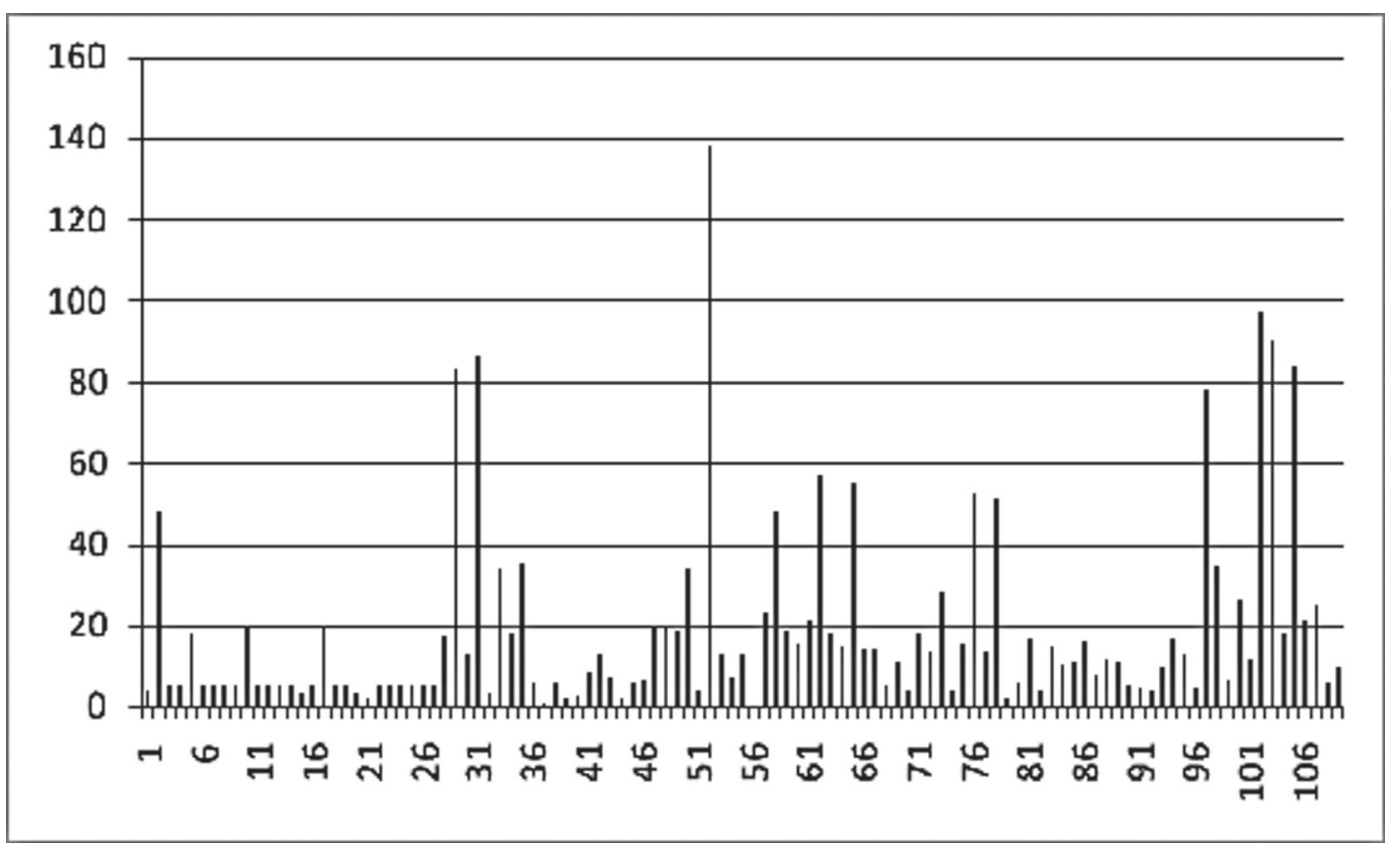

Fig. 4: Profundidades, en km, de los sismos sentidos del año 2010.

\section{SISMICIDAD POR REGIONES}

\section{Guanacaste}

En la región de Guanacaste la actividad sísmica se muestra menor y más dispersa con respecto a las regiones central y sur del país. Se registraron varios sismos en la zona de subducción de la placa del Coco bajo la placa Caribe, en la costa del Pacífico y otros cerca del extremo sureste de la península de Nicoya asociados a fallamiento de rumbo.

Un sismo se reportó frente a las costas de Guanacaste el día 24 de febrero con una magnitud de 5,6 y asociado al proceso de subducción. Otro sismo se produjo el día 27 de abril en San Rafael de Guatuso, este sismo fue de magnitud 5,0 y fue profundo $(138 \mathrm{~km})$ asociado a la subducción por lo que no se sintió muy fuerte, sino leve.

El día 26 de mayo un nuevo sismo fue sentido principalmente en Guanacaste, con una magnitud de 5,1 y asociado a la subducción de la placa del Coco nuevamente. En julio se registró un evento Bijagua de Upala y en Nicoya también se registró otro sismo asociado, en este caso, a la subducción de la placa Coco

Durante el mes de agosto del 2010 se reportó un sismo sentido en el Pacífico Norte, asociado a la subducción de la placa Coco bajo la placa Caribe.

Seis eventos se produjeron en la zona del Pacífico Norte en setiembre, al SE de Cóbano, asociados a un sistema de fallamiento de rumbo y sinestral en el piso oceánico del Golfo de Nicoya en la falla denominada como "falla Barranca". El sismo principal ocurrió el día 16 de setiembre con una magnitud de 5,1 Mw. Este sismo, aunque alarmó a las poblaciones de Cóbano, Paquera, Cabuya y Montezuma, solo produjo daños leves en algunas construcciones y caída de objetos. Esta falla se volvió a reactivar en diciembre con un sismo de 
magnitud 4,1. En la península de Nicoya se registró otro evento el día 26 de setiembre al SE de Mansión (M 4,1) pero asociado a la subducción.

\section{Zona Norte}

El día 8 de octubre se sintió un fuerte temblor en casi todo el territorio nacional y particularmente en el Valle Central. Este sismo tuvo una magnitud de 5,9 (Mw), se ubicó al NE de Zarcero $\mathrm{y}$ fue muy profundo $(84 \mathrm{~km}) \mathrm{y}$ fue seguido por varias réplicas. Esta actividad se relaciona con una ruptura profunda en la placa del Coco y se le denomina sismos intraplaca. Aunque el sismo se sintió fuerte en el Valle Central (Intensidad V) no produjo daños.

\section{Pacífico Central}

En esta región se localizaron 19 de los sismos sentidos. El día 22 enero se ubicó $17 \mathrm{~km}$ al NE de Quepos (M 4,7), sismo profundo relacionado con el proceso de subducción de la placa del Coco bajo la placa Caribe y también otro el día 28 en Carrizal de Alajuela (M 4,3) asociado a la subducción. Otro sismo se presentó el día 8 febrero con una magnitud de 4,4; sismo profundo asociado con el proceso de subducción de la placa del Coco bajo la placa Caribe en la zona de Quepos.

Durante el mes de abril del 2010 se reportaron otros sismos sentidos en el pacífico central, siendo el primer evento del mes el del día $1^{\circ}$ de abril, sismo sentido en forma moderada en la zona del Pacífico central que se ubicó al sur de Playa Dominical y tuvo una magnitud de 5.0. Otros dos sismos de magnitud 4,5 se produjeron esta misma localidad (Pacífico Central) en los días 8 y 11 de abril (Dominical y Parrita). Estos sismos se asociaron a la subducción de la placa del Coco bajo la placa Caribe.

El día 20 de mayo se reportó otros dos sismos localizados al SW de Quepos (M 5,9) y al sur de Parrita (M 4,3) asociados al proceso de subducción de la placa del Coco bajo la placa Caribe. El día 31 de mayo un nuevo sismo en la zona de Quepos (M 5,7) fue sentido en esa zona y el Valle Central, asociado nuevamente a la subducción.
Solamente se reportaron algunos daños en supermercados en Quepos por la caída de mercadería de estantes a raíz del sismo del 20 de mayo.

Durante el mes de junio del 2010 se reportaron dos sismos sentidos ambos en la zona del Pacífico Central. El primer evento del mes fue el del día 21 de junio, sismo sentido en forma moderada en la zona del Pacífico Central y tuvo una magnitud de 3,9. Otro sismo se produjo el día 30 de junio en la misma zona con una magnitud de 3,8 . Estos sismos se asociaron al proceso de subducción de la placa del Coco bajo la placa Caribe. Ambos temblores fueron sentidos en forma moderada en la zona del Pacífico central y no provocaron daños.

Durante el mes de agosto del 2010 se reportaron 2 sismos sentidos en el pacífico central. asociados a la subducción de la placa Coco bajo la placa Caribe. De ellos, los del 6 y 15 de agosto, fueron los de mayor magnitud del mes (Ml 5,0), sin embargo, por ser sismos profundos no produjeron daños.

En el mes de octubre también tuvo lugar otro sismo sentido, se ubicó al SW de Dominical (Mag. 4,2) asociado a la subducción de la placa del Coco.

En la zona de Los Santos dos sismos de magnitudes moderadas $(3,6)$ asociados a fallamiento local se registraron los días 5 y 14 de octubre al SW de San Marcos de Tarrazú. Estos fueron percibidos en forma leve en la zona de los Santos.

\section{Valle Central}

En San Isidro de Heredia se registró un sismo el día 11 de enero que tuvo una magnitud de 3,6 y fue asociado a fallamiento local. El 30 de enero en San Juan de Dios de Desamparados, de magnitud 3,6 asociado a fallamiento y otro al NW de San Isidro de Heredia, sismo profundo asociado al proceso de subducción (M 4,3). No se reportaron daños por los sismos sentidos durante el mes. Otro sismo tuvo lugar el día 5 de febrero y se ubicó en Guácimo de Atenas y tuvo una magnitud de 3,5 y se asocia a fallamiento local.

Durante el mes de marzo del 2010 se reportaron otros eventos, siendo el principal evento del 
mes el del día 5 de marzo, sismo sentido fuerte en San José, que se ubicó en Sabanilla de Montes de Oca y tuvo una magnitud de 4,5. Otro sismo de magnitud 3,5 se produjo en esta misma localidad en día 13 de marzo. Estos sismos se asociaron a la falla Cipreses. Otra actividad sísmica importante tuvo lugar en Pacayas de San Rafael de Oreamuno de Cartago en donde se registró un enjambre sísmico, algunos de esos eventos reportados como sentidos en la zona de Pacayas y Oreamuno, principalmente el día 2 de marzo. Estos sismos tuvieron magnitudes moderadas y se originaron en fallamiento local. El día 30 de abril se registró un nuevo sismo sentido en la zona del volcán Irazú (Mag. 3,6) asociado a fallamiento. Otro sismo de magnitud 4,0 se produjo el día 12 de mayo en el Bajo de la Hondura. Estos sismos se asociaron a fallamiento.

En julio también se registraron sismos en la zona norte de Cartago asociados a fallamiento local y el mes terminó con un sismo por falla local en San Jerónimo de Moravia (Mag. 3,8).

El día 24 de setiembre se reportó otro sismo sentido en el Valle Central; este sismo se ubicó cerca de Tres Ríos, tuvo una magnitud de 4,1 y fue asociado a una fractura profunda en la placa Coco (Fig. 5).

\section{Región Caribe}

Se registró actividad sísmica principalmente en Turrialba, cerca y en los alrededores del macizo del volcán Turrialba. Otra actividad sísmica importante tuvo lugar en la región Caribe con un sismo sentido el día 21 de abril localizado cerca de Guápiles (M 3,5) asociado a fallamiento local.

El día 12 de mayo, se reportó un sismo sentido en forma moderada en la zona del Caribe que se ubicó frente a la costa Caribe de Nicaragua y tuvo una magnitud de 5.0. En Sixaola se reportó un sismo de magnitud 4,6 que se produjo el día 13 de mayo al SW de Sixaola, en el Caribe. Este sismo se asocia a fallamiento.

Cerrando el 2010, el día 29 de diciembre se registró un sismo cerca de la ciudad de Limón el cual tuvo una magnitud de 4,6 y se originó en fallamiento local.

\section{San Isidro de Pérez Zeledón}

El 20 de marzo se reportó un sismo sentido en Santa Eduviges de Pérez Zeledón (Mag. 3,6).

\section{Zona Sur}

Durante el mes de enero del 2010 se reportaron 31 sismos sentidos en el país, siendo la principal actividad del mes en la zona Sur de Costa Rica. Entre los días sábado 23 y el lunes 25 de enero de 2010 , se registraron unos 60 sismos en la zona sur de Costa Rica y el suroeste de Panamá, destacando un total de 26 sismos con magnitudes mayores a 3,8 en la escala Richter, que son los mayormente sentidos por la población de la zona. Estos se ubicaron en la zona de la Península Burica, siendo debidos a procesos tectónicos dentro de la región del Punto Triple de interacción entre las placas, Coco, Nazca y Bloque de Panamá. Entre éstos sobresalen los dos más fuertes, sucedidos el día 23; el primero, ocurrido a las 03:09 am de magnitud local escala Richter 5,0 Ml y el otro a las 08:43 am de magnitud 4,9 Ml. Parte de estos eventos tienen epicentros frente a la costa oeste de la Península Burica y otros ubicados en la parte central de esa Península. El día 29 se registró un sismo relacionado con fallamiento local en Sabalito con magnitud de 4,0.

Otro evento se registró el día 9 de febrero, se ubicó cerca de Laurel, en la zona sur y tuvo una magnitud de 5,1 y se sintió fuerte en dicha zona del país, aunque no causó daños. Durante el mes de julio del 2010 se reportaron 5 eventos que se produjeron en la zona sur del país en Golfito, Pto. Armuelles, Laurel, y San Vito. El mayor de ellos fue el del 29 de julio con una magnitud de 4,4. Todos estos sismos tuvieron intensidades moderadas ( IMMmax. III) y no generaron daños. También en la zona sur, en agosto se registraron dos eventos sentidos al NE de San Vito de Coto Brus, asociados a fallamiento.

En agosto la actividad más importante fue la registrada el día 27, $20 \mathrm{~km}$ al norte de Buenos Aires, provincia de Puntarenas en donde un enjambre sísmico alarmó un poco a las poblaciones vecinas. Los sismos se ubicaron cerca del 
cerro Dúrika, en la parte alta de la cordillera de Talamanca. En setiembre en Buenos Aires de Puntarenas, se registraron 6 réplicas más de la actividad que se inició en esa zona en agosto de 2010. Esta actividad también está asociada a fallamiento local.

Este enjambre sísmico está relacionado con el fallamiento local de tipo normal de las cabeceras del Río Telire, donde en otras ocasiones se ha registrado sismicidad. El análisis de mecanismo focal para los dos principales eventos, indica fallamiento normal, relacionado a fuerzas gravitacionales, posiblemente debidas al los sistemas de fracturas, por el proceso de levantamiento de la Cordillera de Talamanca.

\section{ACTIVIDAD VOLCÁNICA}

\section{Volcán Tenorio}

El volcán Tenorio ha aumentado la actividad sísmica en los últimos años y muy especialmente en el primer semestre del 2010. Además, el día 6 de julio a las 9:44 p.m. se produjo un sismo de magnitud $\mathrm{Mc}=3,4$ a una profundidad de $4,2 \mathrm{~km}$ que fue sentido en Bijagua de Upala y alrededores. Posteriormente se registraron algunas réplicas (24 sismos) que sugieren que se trata de una falla de rumbo N-S. Las profundidades de estos eventos del año 2010, en su gran mayoría, no sobrepasan los $10 \mathrm{~km}$. Esto hace pensar que podemos estar ante el ascenso de magma o por lo menos una transferencia de esfuerzos en la parte superior de la corteza que ya está alcanza fallas locales como la que se activo el pasado 6 de julio.

\section{Volcán Arenal}

La actividad del Arenal durante el mes de mayo 2010 presentó las mismas características de los últimos meses: intensa actividad fumarólica desde la cima, coladas de lavas blocosas cortas con una baja tasa de efusión, que provocan deslizamientos de rocas, y explosiones estrombolianas moderadas, siempre dentro del patrón de normalidad de los últimos 10 años de la actividad, que se prolonga desde julio de 1968 (casi 42 años continuos).

Dado que la lava se mantiene con una baja tasa de extrusión de cerca de $0,1 \mathrm{~m} 3 / \mathrm{s}$ (un tercio de la tasa en la década de los 1990), las coladas (< $1 \mathrm{~km}$ de longitud) se mantienen en las partes del cono de alta pendiente $\left(>35^{\circ}\right)$, desde donde con frecuencia de minutos ocurren deslizamientos de las rocas del frente blocoso de las lavas, y llegan a formar abanicos de bloques sueltos en la parte media del cono (entre 800-1000 m s n.m.). Estos depósitos están muy engrosados (decenas de $\mathrm{m}$ ) entre 1 y $2 \mathrm{~km}$ de distancia del cráter activo.

El lunes 24 de mayo en la mañana empezaron a ocurrir pequeños flujos piroclásticos originados en el frente de colada de lava activo, hacia el sector sur-suroeste del volcán.

La colada de lava estaba entrampada en un obstáculo topográfico de una lava previa, de modo que al superar la barrera topográfica y por efecto de la fuerza de gravedad, los deslizamientos de bloques del frente se tornaron más voluminosos y gradaron sucesivamente a flujos piroclásticos, de los cuales se contaron 15 hasta la 1 de la tarde de ese día. Como resultado, parte de ese sector sufrió una intensa erosión por parte de los flujos.

Posteriormente a esta actividad, el frente de colada de lava se activó y emitió continuos bloques que se transportaron por gravedad ladera abajo, rebotando y rodando hasta depositarse en los abanicos de bloques cerca de los 800-900 m de altitud. Los bloques, con interiores incandescentes por las altas temperaturas (cerca de 1000 ${ }^{\circ} \mathrm{C}$ ), fueron vistos "al rojo vivo" incluso en horas diurnas. Por otra parte, el conducto, al quedar relativamente liberado de la coraza de lava superior, ha permitido una actividad explosiva más intensa, con frecuentes explosiones estrombolianas, que proyectan bloques y cenizas hasta un altura de 200-300 m sobre el cráter. Los bloques caen en 
proyección balística en la periferia del cráter y las cenizas son transportadas por los vientos alisios predominantes, que van principalmente hacia el oeste. No obstante, con la entrada de los vientos del Pacífico, hay también proyección de ceniza hacia el norte y hacia el noreste del volcán.

\section{Volcán Poás}

La temperatura del lago intracratérico se mantuvo en 53 grados Celsius con un grado de acidez de cero (la acidez más extrema posible) durante el año.

El domo ubicado en el centro del cráter presentó temperaturas de más de 600 grados Celsius con un punto máximo de 633 grados. Estas altas temperaturas provocan que el lago este en constante evaporación y que en dos años el nivel haya descendido 20 metros en total. Es por eso que ahora los turistas pueden apreciar desde el mirador estructuras geológicas del cráter que antes no se apreciaban por estar cubiertas por el agua ácida. El lago presenta constantes burbujeos y se aprecian natas de sedimentos y azufre flotando en el lago, proveniente de las piscinas de azufre ubicadas en el fondo del mismo.

Durante el mes de mayo del 2010 se observaron al menos 6 erupciones freáticas provenientes de la Laguna Caliente del volcán Poás. Posiblemente la erupción más fuerte, fue la ocurrida el 29 de mayo del 2010. Esta erupción genero "chorros" de lodo que bañaron parte de las paredes del intracráter, especialmente hacia el sector Sur y Oeste.

\section{Volcán Irazú}

No mostró cambios importantes durante el 2010 salvo un incremento importante en la actividad sísmica de tipo tectónico en las fallas que rodean al macizo, tanto por el norte como por el sur. Muchos de esos sismos fueron reportados como sentidos en la zona cercana al volcán, pero fueron de magnitudes moderadas (menores a 4).

\section{Volcán Turrialba}

En el mes de enero fue importante la actividad que se reportó en el volcán Turrialba la cual se incrementó a partir del día 5 de enero y lanzó gases y alguna ceniza que puso en alerta a las autoridades de la Comisión Nacional de Emergencias (CNE) y a los científicos de la Red Sismológica Nacional (RSN), que incrementaron las medidas de seguridad y el monitoreo más detallado de la evolución de esta actividad.

Enjambres tectónicos con epicentros a lo largo de un rasgo tectónico principal (una zona al SW con cráteres, fallas, conos piroclásticos y fumarolas) fueron registrados durante todo el año. Entre los principales focos de actividad sísmica que se han detectado destacan principalmente un grupo grande de sismos al NE del macizo del Turrialba, otro al norte del volcán Irazú, probablemente relacionado con la falla Río Sucio y otras aledañas y otro grupo por el sector sur, SE y SW del Irazú, por las localidades de Cot y Pacayas de Cartago en donde se localizan varias fallas pequeñas y también otros sismos cerca del cráter del Turrialba y hacia el sector sur-suroeste del mismo, que coinciden con la falla Ariete.

La lluvia ácida $(\mathrm{pH}<5)$ ha afectado notablemente $\sim 50 \mathrm{~km}^{2}$ en el flanco occidental. El 5-6 de enero del 2010, ceniza lítica fue eruptada debido a una actividad fumarólica-freática fuerte de un nueva abertura en el sector sur de la pared interna del cráter suroeste. Las cenizas muy finas se distribuyeron al suroeste y el oeste, alcanzando como trazas de polvo de roca hasta poblaciones de las afueras orientales de San José (hasta a 35 km desde el volcán).

Futuras erupciones (que podrían ocurrir en un plazo de meses a décadas), freáticas o magmáticas, representan una amenaza mayor para granjas y albergues a $5 \mathrm{~km}$ alrededor del volcán 
y amenazas significativas a la agricultura, los potreros y las telecomunicaciones y líneas vitales (viales y eléctricas) en el lado occidental del volcán, hasta varias decenas de kilómetros. La erupción más grande probable afectaría hasta 1,5 millones de personas y el transporte aéreo, porque el principal aeropuerto internacional costarricense está dentro de la región de dispersión de ceniza.

\section{AGRADECIMIENTOS}

Se agradece la valiosa colaboración de todo el personal técnico y profesional de la RSN que mantiene en operación los equipos y procesa toda la información sismológica y vulcanológica que se registra los 365 días del año en Costa Rica. 